\title{
Segmentando residentes según sus actitudes: Revisión de la literatura
}

\author{
José Ramón Cardona* Antoni Serra Cantallops** \\ Universitat de les Illes Balears (España)
}

\begin{abstract}
Resumen: En las últimas décadas, varios estudios empíricos han segmentado las actitudes de los residentes hacia el desarrollo turístico. Se ha realizado una revisión y análisis de estos estudios. Los estudios revisados se llevaron a cabo utilizando metodologías ligeramente diferentes y se encontraron ciertas variaciones en la segmentación resultante. Sin embargo, pueden ser observadas algunas similitudes: ciertos patrones que se repiten. Esto nos lleva a especular que podría haber tipologías predefinidas de residentes cuyo peso en la comunidad de acogida dependerá del destino concreto analizado. Nuestro objetivo es el de establecer una segmentación teórica aplicable a todos los destinos turísticos. Esta segmentación resume la literatura existente y proporciona algunas indicaciones generales a los gestores, en ausencia de estudios específicos y detallados.
\end{abstract}

Palabras Clave: segmentación, actitudes de los residentes, gestión de destinos, planificación del turismo, grupos.

\section{Segmenting residents by their attitudes: literature review}

Abstract: In the last decades, several empirical studies have segmented the attitudes of residents towards tourism development. There has been a review and analysis of these studies. The studies reviewed were carried out using slightly different methodologies and certain variations were found in the resulting segmentation. However, some similarities can be observed: certain patterns that repeat themselves. This leads us to speculate that there might be predefined typologies of residents whose weight in the host community will depend on the particular destination analyzed. Our goal is to establish a theoretical segmentation applies to all destinations. This segmentation summarizes the existing literature and provides some general indications to managers, in the absence of specific and detailed studies.

Keywords: segmentation, resident attitudes, destination management, tourism planning, clusters.

\section{Introducción}

El objetivo de la segmentación es describir una población en relación a determinados fenómenos mediante su congregación en grupos homogéneos y analizando sus respuestas promedio (Williams y Lawson, 2001). Habitualmente los estudios sobre las actitudes de los residentes consideran a la población local como una masa homogénea y se centran en ver como varía la respuesta media bajo el efecto de diversas variables consideradas con capacidad para alterar esta respuesta (Allen, Hafer, Long y Perdue,

1993; Allen, Long, Perdue y Kieselbach, 1988; Besculides, Lee y McCormick, 2002; Gursoy, Chen y Yoon, 2000; Gursoy, Jurowski y Uysal, 2002; Mason y Cheyne, 2000; Perdue, Long y Allen, 1990; Teye, Sirakaya y Sönmez, 2002). Pero es lógico y razonable esperar que exista una variabilidad en las actitudes de los residentes. Existen modelos que buscan describir la evolución de las actitudes de los residentes, como el de Doxey (1975) y el de Butler (1980). Pero en estos casos se considera que las actitudes son homogeneas en un momento dado y variables en el tiempo.

\footnotetext{
E-mail: jose.ramon@uib.es

** E-mail: antoni.serra@uib.es
} 
Cuando observamos la realidad, resulta esperable la existencia de actitudes y percepciones distintas y opuestas entre los residentes (Pi-Sunyer, 1977) en un momento dado, y las acciones para estimular el crecimiento turístico por parte de las administraciones deben tenerlo en cuenta ya que pueden agravar estas diferencias (Brownrigg y Greig, 1976). Esta variabilidad de la sociedad local convierte en una herramienta importante para la gestión del destino turístico la segmentación de los residentes según sus actitudes y percepciones. Cada grupo o segmento de residentes, o sub-comunidad, presentará intereses comunes y propios de su grupo, mostrando costes o beneficios, preocupaciones, etc. que afectan a su actitud hacia el turista (Díaz, Gutiérrez y Garau, 2007). La segmentación es una aproximación factible a la verdadera situación ideal, que es el análisis de las actitudes concretas de cada individuo.

En las últimas décadas se han realizado diversos estudios empíricos que pretenden segmentar los residentes de un destino concreto, con la finalidad de detectar grupos distintos de residentes según su actitud hacia el desarrollo turístico de la región y proceder a caracterizarlos por sus variables demográficas y socioeconómicas. Estas segmentaciones buscan constituir una información fundamental para los gestores de los destinos estudiados. El objetivo que pretendemos es revisar los estudios existentes y establecer una segmentación teórica aplicable en todos los destinos turísticos. Para ello se definen diversos grupos y se establece la variabilidad que poseerán según las características del destino concreto al que se aplique. Esta segmentación teórica permite resumir la bibliografía existente y aporta unas indicaciones generales a los gestores de los destinos turísticos a falta de estudios específicos y pormenorizados para cada destino.

\section{Segmentaciones empíricas}

Diversos autores (Allen, Hafer, Long y Perdue, 1993; Gutiérrez y Díaz, 2006; Jurowski, Uysal y Williams, 1997; Keogh, 1990; Lankford y Howard, 1994; Um y Crompton, 1987) respaldan que el residente está predispuesto positivamente ante modelos turísticos necesarios para el desarrollo económico de una comunidad. Aunque, se determinan variaciones en la predisposición si la población residente es segmentada a partir de criterios intrínsecos o extrínsecos (Lankford, 1994; Murphy, 1985), observando diferencias en la intensidad y dirección de los impactos (beneficios y costes) por segmentos poblacionales (Díaz, Gutiérrez y Garau, 2007; Gutiérrez y Díaz, 2006).

Los diversos estudios en que se procede a la segmentación de los residentes encuentran como elementos definitorios de los segmentos factores que son importantes determinantes de las actitudes de los residentes. Ejemplos de ello son las características demográficas y socioeconómicas, el volumen y tipo de turistas (Brougham y Butler, 1981), el tiempo de residencia en la región, si es nativo o no (Ryan y Montgomery, 1994), etc. Distintos turistas generan distintos impactos y por tanto distintas percepciones y actitudes de los residentes (Brougham y Butler, 1981; Schewe y Calantone, 1978). Destaca el grado de exposición a los turistas como importante factor explicativo de las opiniones de los residentes (Brougham y Butler, 1981).

Uno de las primeras segmentaciones de las actitudes de los residentes la encontramos en un estudio realizado en la peninsula de Sleat (Isla de Skye), en Escocia (Brougham, 1978; Brougham y Butler, 1977; Brougham y Butler, 1981), con una muestra de 123 individuos. En este caso se analizan las actitudes respecto de diferentes impactos socioculturales y debido a la reducida muestra tomada en consideración, y al método empleado, sólo detecta un grupo favorable y un grupo desfavorable en los distintos parámetros estudiados.

Desde entonces, diversos estudios han buscado determinar la segmentación de diversos destinos. En estos estudios se repiten algunos elementos y difieren otros a causa de las diferencias entre destinos y a la metodología aplicada en su realización. A continuación se describen algunos de los más conocidos.

\section{Estados Unidos}

En el trabajo de Davis, Allen y Cosenza (1988) en Florida se identificaron cinco grupos: los "Lovers" (20\%) poseían una imagen extremadamente positiva del turismo y casi no tenían opiniones negativas; los "Haters" (16\%) se caracterizaban por poseer opiniones radicalmente negativas del turismo y los turistas, siendo la Némesis del anterior grupo; los "Cautious Romantics" (20\%) se diferencian de los "Lovers" en que, aunque ambos poseen una opinión positiva, están de acuerdo con algunas apreciaciones negativas acerca del desarrollo turístico; los "In-Betweeners" (18\%) tienen opiniones más moderadas que los "Lovers" y los "Haters" y son parecidos a los "Cautious Romantics" pero son más favorables al crecimiento del sector turístico, y los "Love "em for a Reason" (26\%) son favorables al turismo pero 
no tanto como los "Lovers", además en su caso el apoyo es debido a los beneficios económicos. En este estudio se encontró que muchos de los "Haters" eran nacidos en Florida y tenían un bajo nivel de conocimiento del sector y muchos de los "Lovers" no eran nacidos en Florida y poseían un alto nivel de conocimiento sobre el sector turístico.

En el estudio de Madrigal (1995) se compararon dos destinos, uno rural (Sedona, estado de Arizona), con turismo basado en la naturaleza y la artesanía, con un destino urbano (York, en Reino Unido). En ambos destinos fueron determinados los mismos tres grupos: los "Haters" (31\%) están de acuerdo con los impactos negativos y en desacuerdo con los positivos; los "Lovers" (13\%) están de acuerdo con los impactos positivos y en desacuerdo con los negativos, y los "Realists" (56\%) están de acuerdo con ambos impactos. Los "Realists" son la "mayoría silenciosa", no muestran interés en participar en debates sobre la planificación futura del destino pero son mayoritarios y poseen una visión menos parcial de la situación.

Este estudio encuentra mayores diferencias entre los grupos que entre las ciudades (la pertenencia a un grupo explica el $31 \%$ de la varianza y la ciudad el $2 \%$ ). Los residentes de Sedona tienen una mayor dependencia económica del turismo, habiendo un $9 \%$ más de "Realists" que en York, y los residentes de York residen en la ciudad desde hace mucho más tiempo y son nativos mayoritariamente (99\% de población nativa en York y 1\% en Sedona), habiendo un 17\% más de "Haters" que en Sedona, al poseer mayor proporción de población nativa (Madrigal, 1995). En diversos estudios se ha detectado que la población nativa tiene una postura más negativa hacia el desarrollo turístico (Canan y Hennessy, 1989; Davis, Allen y Cosenza, 1988; Um y Crompton, 1987). Los clústers detectados en el estudio de Madrigal (1995) probablemente deban su composición al hecho de que los dos destinos estudiados, Sedona y York, reciben tres millones de visitantes al año cada uno con poblaciones residentes de 7.720 y 100.000 habitantes respectivamente.

En otros estudios, Canan y Hennessy (1989) encontraron sustanciales diferencias entre los residentes agrupados según su actitud hacia el desarrollo turístico en Moloka'i (Hawaii). Schroeder (1992) detecto tres grupos en su estudio de Flagstaff (Arizona). Los residentes favorables al desarrollo turístico eran el $35 \%$ del total y los residentes con posturas opuestas o neutrales representaban el 51\%. Martin (1995) encontró cuatro grupos en Teton Country (Montana), el 59\% favorable al turismo y el 13\% contrario. El resto de grupos tendían a la neutralidad.

\section{Reino Unido}

El trabajo de Ryan y Montgomery (1994) en Bakewell (Inglaterra) se basa en el de Davis, Allen y Cosenza (1988) en Florida y usa una muestra muy reducida. Tres grupos fueron identificados en el estudio de Bakewell: los "Enthusiast" (22\%) apoyan el turismo pero no excesivamente; los "Somewhat Irritated" (24\%) tienen opiniones negativas acerca de los impactos del turismo y son escépticos hacia sus beneficios; y los "Middle-of-the-Roaders" (54\%) que se encuentran entre los otros dos grupos.

\section{Australia}

En un estudio referente a un acontecimiento, The Gold Coast IndyCar Race, se determinaron cinco grupos de residentes en relación a su actitud hacia el evento en cuestión (Fredline y Faulkner, 2000): los "Ambivalent Supporter (Cautious Romantics)" (29\%) poseen respuestas muy ambivalentes y tienden a posturas moderadas o a responder neutro; los "Haters" (15\%) se caracterizan por un elevado nivel de desacuerdo con los puntos referentes a los beneficios que aporta el evento y un gran nivel de acuerdo con los impactos negativos; los "Realists"(24\%) se encuentran de acuerdo con impactos tanto positivos como negativos; los "Lovers" (23\%) son defensores entusiastas del evento, y los "Concerned for a Reason" $(9 \%)$ se caracterizan por estar preocupados por algunos efectos negativos.

Tamborine Mountain (Gold Coast) se puede considerar en la fase de desarrollo del modelo de ciclo de vida descrito por Butler (1980). En el estudio de Weaver y Lawton (2001) se detectaron tres grupos: los "Supporters" (27\%) asocian el sector con fuertes beneficios económicos y sociales para la comunidad, son los últimos en llegar y trabajan en el sector; los "Opponents" (22\%) son personas con muchos años de residencia en la región, tienen un menor contacto con los turistas y son los menos predispuestos hacia el desarrollo turístico, y los "Neutrals (51\%) reconocen las ventajas pero también algunas desventajas sociales, aunque su valoración global es favorable.

\section{Nueva Zelanda}

En el estudio de Evans (1993) se identificaron cuatro grupos: "Lovers", "Haters", "Controlled" y "Selfish". Los "Lovers" (20\%) son muy favorables al turismo y demográficamente se caracterizan por tener un alto nivel de renta, estar casados, poseer trabajo, pertenecer a los Pakeha (neozelandeses descendientes de europeos) y ser de mediana edad. Los "Haters" (11\%) poseen una actitud fuertemente contraria al 
turismo, son diferentes de los "Lovers" en casi todo y tienen una amplia proporción de personas viudas, divorciadas o separadas, personas sin empleo o retirados, no-Pakeha (Maoris o grupos étnicos del Pacífico), y hay más mujeres que hombres. Los "Controlled" (32\%) son similares a los "Lovers" pero reconocen tanto los costes como los beneficios, sus valoraciones son más moderadas que las de los "Lovers" y los "Haters", no les entusiasman los cambios y demográficamente son mayoritarios los casados, retirados o con empleo, Pakeha y las mujeres. Los "Selfish" (37\%) están de acuerdo con los impactos positivos y negativos del turismo, desean que aumente el ocio nocturno, los casinos y la inversión extranjera, ven el turismo como un medio para mejorar su situación personal, y demográficamente se caracterizan por ser, mayoritariamente, hombres, jóvenes, solteros, estudiantes y no-Pakeha.

Ryan, Scotland, y Montgomery (1998) compararon Rangitikei (New Zealand) y Bakewell (United Kingdom). Partiendo de una muestra de 176 encuestas realizadas en Rangitikei se obtuvieron tres grupos de residentes. Los "Moderate Enthusiasts" (42,5\%) apoyan el turismo. Los "Extreme Enthusiast" $(17,5 \%)$ poseen un fuerte apoyo hacia el turismo y se oponen a la planificación si implica una restricción del crecimiento. Los "Cautious Supporters" (40\%) son partidarios del desarrollo turístico pero poseen las puntuaciones más bajas al valorar los impactos positivos y les preocupa mucho la planificación turística.

El trabajo de Williams y Lawson (2001) analizó las actitudes de los residentes en diez ciudades de Nueva Zelanda (Auckland, Blenheim, Christchurch, Hokitika, Kaikoura, Napier, Queenstown, Rotorua, Taupo y Whangarei). En su estudio detectaron cuatro grupos de residentes según su actitud hacia el turismo: Lovers, Cynics, Taxpayers y Innocents. Los "Lovers" (45\%) son los que más aprueban el turismo y demográficamente se caracterizan por tener mayor proporción de personas casadas, con casa propia y elevadas rentas. Los "Cynics" (10\%) son los que menos aprueban el turismo y demográficamente se caracterizan por ser más viejos, tener menos renta, tener una menor proporción de personas casadas y de propietarios de viviendas que el resto de la muestra. Los "Taxpayers" (25\%) sólo muestran una mayor preocupación por los factores de carácter económico y por los servicios públicos, viven en la población desde hace menos de dos años, consideran que el turismo provoca importantes cambios en los valores y estilos de vida, y demográficamente se caracterizan por una mayor proporción de separados, divorciados y propietarios de casas. Los "Innocents" (20\%) no perciben los beneficios del turismo al tener escaso contacto con la esfera turística.

Thyne y Lawson (2001) realizaron un estudio de Southern Lakes Region (Alexandra, Cromwell, Clyde, Omarama, Twizel, Arrowtown, Otematata, Queenstown, Te Anau y Wanaka) con una muestra de 1094 encuestas válidas y obtuvieron 4 grupos: Lovers, We Miss Out, Self-Interest Supporters y Critics. Los "Lovers" $(14,4 \%)$ son los más favorables hacia el turismo, gustándoles todo del turismo y no viendo ningún impacto negativo. Los "We Miss Out" (39,6\%) sienten que no son participes de los beneficios del turismo. Son los más contrarios a considerar que el turismo ha generado mejoras en la oferta recreativa y comercial, en las oportunidades de empleo y en los servicios públicos. Los "Self-Interest Supporters $(29,7 \%)$ apoyan el turismo por los beneficios personales que les reporta. Consideran que ha mejorado la oferta comercial y de ocio, los servicios públicos y las oportunidades de empleo. Son personas jóvenes con bajos salarios y poca formación. Los "Critics" $(16,3 \%)$ consideran que el turismo no aporta beneficios y son el único grupo contrario a un aumento en el número de turistas, preferirían que se redujera. Solo reconocen los impactos negativos del turismo y ninguno de los positivos.

Los "Haters" de Evans (1993), los "Cynics" de Williams y Lawson (2001) y los "Critics" de Thyne y Lawson (2001) sugieren que las personas con una peor valoración de la actividad turística tienen un menor nivel de satisfacción con su vida en general. Es decir, la gente con una vida en pésimas condiciones es más negativa en sus valoraciones de distintos elementos, sean acciones de marketing (Lawson y Todd, 1997) o de otro tipo.

\section{Grecia}

En un estudio realizado en Heraklio, Chania, Rethymno y Agios Nikolaos (costa norte de Creta) con una muestra de 194 hogares, Andriotis y Vaughan (2003) detectaron tres grupos. Los "Advocates" (42,5\%) poseen la postura más favorable hacia el turismo y consideran el turismo fundamental para el bienestar de la isla. Los "Socially and Environmentally Concerned" (39,5\%) poseen las visiones más negativas hacia los impactos socioculturales y medioambientales del turismo. Los "Economic skeptics" (18\%) poseen las visiones más negativas hacia los impactos económicos. La segmentación resulta de difícil interpretación y análisis poco claro, no resultando comparable con otros estudios similares (Andriotis y Vaughan, 2003).

\section{España}

En los últimos diez años se han iniciado estudios sobre las actitudes de los residentes en las islas Baleares. El primero de los cuales es Anàlisis de les actituds i percepcions de la població resident 
enfront del desenvolupament turístic a Balears dirigido por el Dr. Antoni Serra en 2003 y financiado por el CITTIB. Posteriormente se han realizado algunos estudios más. En el estudio de Aguiló y Rosselló (2005) encontraron cinco grupos.

- Los "Development Supporters" (11\%) son los que creen con más firmeza que gracias al turismo existen más oportunidades de trabajo y de negocio y que el balance global del turismo es positivo. Su perfil sociodemográfico presenta una ligera tendencia a pertenecer a hogares con trabajadores en el sector, hijos menores y de ingresos medios altos.

- Los "Prudent Developers" (26\%) creen que gracias al turismo existen más oportunidades de trabajo, si bien reconocen que el turismo ha generado un cambio importante en la cultura local. A diferencia del grupo anterior son especialmente conscientes de los efectos nocivos del turismo. Su perfil sociodemográfico se caracteriza por unos ingresos menores que la media y menos vinculados al turismo que el resto. Además tienen gran apego a la comunidad a pesar de ser mayoritariamente personas nacidas en el resto de España.

- Los "Ambivalent and Cautious" (24\%) presentan una opinión bastante neutral a la hora de valorar los efectos positivos y negativos del turismo, y aunque otorgan un aprobado al balance entre ganancias y costes debidos al turismo su valoración está por debajo de la media. Su perfil sociodemográfico muestra una ligera tendencia a que en este grupo se integren nativos residentes en Palma que suelen sentirse menos integrados y más dispuestos a cambiar de domicilio.

- Los "Protectionists" (20\%) son los que más énfasis ponen en los aspectos negativos del turismo y creen que el balance entre los aspectos positivos y negativos es negativo. Su perfil sociodemográfico muestra a individuos integrados en su municipio, muy preocupados por el medio ambiente y poco preocupados por el desarrollo económico. Tienen ingresos menos dependientes del turismo que el resto y son individuos nacidos en las islas y con ingresos medio-altos.

- Los "Alternative Developers" (18\%) creen con más determinación que el resto que el turismo ha aumentado las posibilidades de trabajo para los residentes y no valoran tan negativamente los aspectos negativos ligados al turismo. Su perfil sociodemográfico muestra a individuos que no suelen haber nacido en las islas y sí en un país extranjero, y que poseen ingreso medios altos. En general no se sienten tan arraigados como el resto y poseen menos preocupación que el resto por la inseguridad ciudadana y el desarrollo económico.

En el estudio de Gutiérrez y Díaz (2006), con una muestra de 651 encuestas realizadas en Tenerife (Islas Canarias) en 2004 y 2005, se diferencian tres grupos de residentes según su actitud hacia el turismo, parecidos a los grupos determinados por Ryan y Montgomery (1994): ambiguos, anti-desarrollo y pro-desarrollo. Los ambiguos, "normalizados". Este segmento se caracteriza por considerar que la actividad favorece la economía de la comunidad, aunque no cree que se logren efectos positivos muy destacados sobre la dimensión social, cultural y aún menos medioambiental, donde destacan los costes. Los anti-desarrollo turístico, "radicales y sensibles a los impactos de la actividad turística". Este segmento se caracteriza por considerar que la actividad turística favorece la economía de la comunidad, pero la rechaza y cree que es fuente importante de impactos negativos (sociales, culturales y medioambientales). En este segmento se encuentran los jóvenes menores de 25 años que no viven ni trabajan del turismo. Los pro-desarrollo turístico, "sensibles a los impactos positivos de la actividad turística". En este segmento nos encontramos con los residentes que consideran a la actividad turística como fuente de impactos positivos en todas las dimensiones, entre las que destaca la económica. Destacan las personas de edad madura, que viven y trabajan en zonas turísticas, con baja cualificación y formación. En el estudio de Gutiérrez y Díaz (2006) se observa que existe acuerdo general entre los diferentes segmentos de residentes en relación con el impacto económico del sector turístico, en sentido positivo.

En el estudio de Díaz, Gutiérrez y Garau (2007) se compararon dos destinos turísticos con similitudes: Tenerife (Islas Canarias) y Mallorca (Islas Baleares). En ambos casos se trata de islas con fuerte presencia turística y con comunidades con un elevado nivel de arraigo e identidad con su realidad social y cultural. Con una muestra de 487 encuestas en Tenerife y 584 encuestas en Mallorca, realizadas en 2006, se diferencian tres grupos de residentes, según su actitud expresada hacia el turismo, parecidos a los grupos determinados por Gutiérrez y Díaz (2006) o por Ryan y Montgomery (1994): Positivos, Cautos y Críticos. Los Positivos poseen una predisposición positiva hacia el turismo como actividad que fomenta los beneficios económicos, sociales y culturales. Creen que la actividad turística no es responsable de los costes de las diferentes dimensiones, a excepción de la dimensión medioambiental donde considera los efectos negativos que produce la actividad turística. Los Cautos no son extremistas y 
valoran poco importantes los beneficios y costes de las diferentes dimensiones, no consideran la actividad turística culpable de los males, aunque tampoco cree que de lugar a beneficios notables. Consideran que el turismo es una actividad económica necesaria. Los Críticos poseen valoraciones contrarias a las del primero valorando siempre como poco importantes los beneficios y amplificando los costes de las dimensiones social, cultural y mediambiental. Este segmento considera que la actividad es responsable de los diversos costes económicos, sociales, culturales y por supuesto medioambientales considerados en el análisis, aunque valoran positivamente el impacto en el bienestar económico de la comunidad.

El factor "oportunidad de empleo" no discrimina entre segmentos en ninguna de las dos islas. La explicación es que todo el mundo esta de acuerdo con que el turismo genera oportunidades de empleo, aunque luego haya matizaciones en cuanto a la calidad de este empleo. Al igual que en el estudio de Madrigal (1995), se observan mayores diferencias entre los segmentos que entre las islas. En cuanto a las diferencias entre destinos se percibe que determinados impactos difieren en intensidad, incidiendo sobre la predisposición hacia la actividad turística de manera diferente. En Mallorca la opinión no es tan positiva, un $84 \%$ de los encuestados consideran beneficioso el turismo, mientras que en Tenerife la cifra asciende al 93\%. En Mallorca el factor que incide sobre la valoración de los impactos es la permanente influencia del visitante en un espacio temporal reducido (estacionalidad), en todo el territorio y con una presión sobre el residente muy superior a la de Tenerife, 12,1 frente a 6,4.

Italia

Brida, Osti y Barquet (2010) detectaron en su estudio de la pequeña comunidad de Folgaria (Trentino-South Tyrol) cuatro grupos distintos: Environmental Supporters, Development Supporters, Protectionist y Ambivalent. Los "Environmental Supporters" (40\%) estan parcialmente de acuerdo con los impactos positivos económicos y socioculturales, y con los impactos negativos medioambientales. Además, estan parcialmete en desacuerdo con los impactos negativos socioculturales y los impactos positivos medioambientales. Los "Development Supporters" (27\%) están de acuerdo con los impactos positivos del turismo y en desacuerdo con los impactos negativos. Este grupo está caracterizado por: mayoritariamente son hombres, viven en la región desde hace 35 años de media y un miembro de la unidad familiar trabaja en el sector desde hace más de cinco años. Los "Protectionists" (14\%) están de acuerdo con los impactos negativos en el medioambiente y en desacuerdo con los impactos positivo en la región. No tienen postura clara en relación a los impactos socioculturales. Son mayoría los hombres y ningún miembro de la familia trabaja en el sector. Los "Ambivalents" (18\%) están de acuerdo con los impactos positivos económicos y tienen una postura ambigua en relación a los impactos socioeconómicos y medioambientales. Este grupo está formado mayoritariamente por hombres que se encuentran cerca de la edad de jubilación, son los que llevan más tiempo viviendo en la región y ningún miembro de la unidad familiar trabaja en el sector.

\section{Paralelismos entre los estudios empíricos}

Los diversos análisis clúster revisados se caracterizan por usar metodologías ligeramente distintas en destinos distintos. Aún así, se pueden observar algunas similitudes en el resultado obtenido. Salvo en dos estudios que muestran carencias (Andriotis y Vaughan, 2003; Ryan, Scotland y Montgomery, 1998), se detecta siempre la presencia de dos grupos: los defensores del turismo y los detractores a ultranza. Ambos grupos aparecen siempre, aunque con proporciones distintas.

Los partidarios representan porcentajes de la población que oscilan entre el $11 \%$ en los destinos maduros y masificados y el $45 \%$ en las áreas que se encuentran en sus fases iniciales. Son los "Lovers" de Davis, Allen y Cosenza (1988), Evans (1993), Fredline y Faulkner (2000), Madrigal (2005), Thyne y Lawson (2001), y Williams y Lawson (2001), los "Enthusiast" de Ryan y Montgomery, los "Supporters" de Weaver y Lawton (2001), los "Development Supporters" de Aguiló y Rosselló (2005) y Brida, Osti y Barquet (2010), etc. Este grupo se caracteriza por dar enorme importancia a los impactos positivos generados por el sector y minimizar los impactos negativos. Además reciben o esperan recibir importantes beneficios del turismo.

Los detractores representan porcentajes que oscilan entre el $11 \%$ en los destinos incipientes y el $31 \%$ en los destinos maduros y masificados. Son los "Haters" de Davis, Allen y Cosenza (1988), Evans (1993), Fredline y Faulkner (2000), y Madrigal (2005), los "Somewhat Irritated" de Ryan y Montgomery (1994), los "Opponents" de Weaver y Lawton (2001), los "Cynics" de Williams y Lawton (2001), los "Critics" de Thyne y Lawson (2001), los "protectionists" de Aguiló y Rosselló (2005) y Brida, Osti y Barquet (2010), etc. Este grupo da enorme importancia a los impactos negativos y no consideran que reciban ningún beneficio del sector pero si costes. En general, preferirían que el desarrollo turístico fuera menor o incluso nulo. 
Cuadro 1: Comparativa entre los principales estudios

\begin{tabular}{|c|c|c|c|c|c|}
\hline & Críticos & $\begin{array}{l}\text { Sin Opinión } \\
\text { Formada }\end{array}$ & $\begin{array}{c}\text { Partidarios } \\
\text { con Opinión } \\
\text { Matizada }\end{array}$ & $\begin{array}{l}\text { Partidarios } \\
\text { Interesados }\end{array}$ & $\begin{array}{l}\text { Partidarios } \\
\text { Entusiastas }\end{array}$ \\
\hline $\begin{array}{l}\text { Davis, Allen y } \\
\text { Cosenza (1988) }\end{array}$ & Haters (16\%) & $\begin{array}{c}\text { In-betweeners } \\
(18 \%)\end{array}$ & $\begin{array}{c}\text { Cautious } \\
\text { Romantics }(20 \%) \\
\end{array}$ & $\begin{array}{l}\text { Love'em for a } \\
\text { Reason }(26 \%)\end{array}$ & Lovers (20\%) \\
\hline Evans (1993) & Haters (11\%) & & Controlled (32\%) & Selfish (37\%) & Lovers $(20 \%)$ \\
\hline $\begin{array}{l}\text { Ryan y } \\
\text { Montgomery } \\
\text { (1994) }\end{array}$ & $\begin{array}{l}\text { Somewhat } \\
\text { Irritated } \\
(24 \%)\end{array}$ & $\begin{array}{l}\text { Middle-of-the- } \\
\text {-Roaders (54\%) }\end{array}$ & & & $\begin{array}{l}\text { Enthusiast } \\
\quad(22 \%)\end{array}$ \\
\hline Madrigal (1995) & Haters (31\%) & & Realists (56\%) & & Lovers (13\%) \\
\hline $\begin{array}{l}\text { Ryan, Scotland } \\
\text { y Montgomery } \\
\text { (1998) }\end{array}$ & & & $\begin{array}{c}\text { Cautious } \\
\text { Supporter }(40 \%)\end{array}$ & $\begin{array}{c}\text { Moderate } \\
\text { Enthusiast } \\
(42.5 \%)\end{array}$ & $\begin{array}{c}\text { Extreme } \\
\text { Enthusiast } \\
(17.5 \%)\end{array}$ \\
\hline $\begin{array}{l}\text { Fredline y } \\
\text { Faulkner (2000) }\end{array}$ & Haters $(15 \%)$ & $\begin{array}{l}\text { Ambivalent } \\
\text { Supporters } \\
(29 \%)\end{array}$ & Realists (24\%) & $\begin{array}{l}\text { Concerned for } \\
\text { a Reason }(9 \%)\end{array}$ & Lovers (23\%) \\
\hline $\begin{array}{l}\text { Weaver y } \\
\text { Lawton (2001) }\end{array}$ & $\begin{array}{l}\text { Opponents } \\
(22 \%)\end{array}$ & Neutrals (51\%) & & & $\begin{array}{c}\text { Supporters } \\
(27 \%)\end{array}$ \\
\hline $\begin{array}{l}\text { Williams y } \\
\text { Lawson (2001) }\end{array}$ & Cynics (10\%) & $\begin{array}{l}\text { Innocents } \\
(20 \%)\end{array}$ & & $\begin{array}{l}\text { Taxpayers } \\
(25 \%)\end{array}$ & Lovers (45\%) \\
\hline $\begin{array}{l}\text { Thyne y } \\
\text { Lawson (2001) }\end{array}$ & Critics (16\%) & $\begin{array}{l}\text { We Miss Out } \\
(40 \%)\end{array}$ & & $\begin{array}{c}\text { Self-Interest } \\
\text { Supporters } \\
(30 \%)\end{array}$ & Lovers (14\%) \\
\hline $\begin{array}{l}\text { Andriotis y } \\
\text { Vaughan (2003) }\end{array}$ & & & \begin{tabular}{|c|} 
Socially and \\
Environmentally \\
Concerned (40\%) \\
\end{tabular} & $\begin{array}{c}\text { Economic } \\
\text { Skeptics (18\%) }\end{array}$ & $\begin{array}{l}\text { Advocates } \\
\quad(42 \%)\end{array}$ \\
\hline $\begin{array}{l}\text { Aguiló y } \\
\text { Rosselló (2005) }\end{array}$ & $\begin{array}{l}\text { Protectionists } \\
(20 \%)\end{array}$ & $\begin{array}{c}\text { Ambivalent } \\
\text { and Cautious } \\
(24 \%)\end{array}$ & $\begin{array}{c}\text { Prudent } \\
\text { Developers (26\%) }\end{array}$ & $\begin{array}{l}\text { Alternative } \\
\text { Developers } \\
\quad(18 \%)\end{array}$ & $\begin{array}{l}\text { Development } \\
\text { Supporters } \\
(11 \%)\end{array}$ \\
\hline $\begin{array}{l}\text { Gutiérrez y } \\
\text { Díaz (2006) }\end{array}$ & $\begin{array}{c}\text { Anti- } \\
\text {-desarrollo } \\
\text { turístico }\end{array}$ & Ambiguos & & & $\begin{array}{l}\text { Pro-desarrollo } \\
\text { turístico }\end{array}$ \\
\hline $\begin{array}{l}\text { Díaz, Gutiérrez } \\
\text { y Garau (2007) }\end{array}$ & Críticos & Cautos & & & Positivos \\
\hline $\begin{array}{l}\text { Brida, Osti y } \\
\text { Barquet (2010) }\end{array}$ & $\begin{array}{l}\text { Protectionists } \\
(14 \%)\end{array}$ & $\begin{array}{l}\text { Ambivalent } \\
(19 \%)\end{array}$ & $\begin{array}{c}\text { Environmental } \\
\text { Supporters (40\%) }\end{array}$ & & $\begin{array}{l}\text { Development } \\
\text { Supporters } \\
(27 \%)\end{array}$ \\
\hline
\end{tabular}

Fuente: Elaboración propia

A parte de estos dos grupos, siempre presentes, los diversos estudios detectan entre uno y tres grupos más de difícil equiparación entre sí. Los paralelismos son difíciles de realizar debido a las variaciones en la metodología usada. Por ejemplo, el conjunto de variables usadas en cada estudio difiere bastante (entre ocho y 62 variables), los estadísticos usados son similares pero no iguales, etc. (Fredline y Faulkner, 2000). Además las regiones estudiadas poseen importantes diferencias. Estos grupos pueden observarse en el Cuadro 1, con sus equivalencias aproximadas. Estos suelen estar caracterizados por poseer una postura general hacia el turismo y su desarrollo menos clara que en los dos grupos anteriores aunque mayoritariamente tienden hacia posturas generales positivas.

En los destinos turísticos muy consolidados y masificados los individuos con alta percepción de los impactos tanto positivos como negativos y que reconocen la dependencia que posee la región del sector suelen ser uno de los grupos más importantes en volumen. Son en sí mismos la constatación de que la población residente 
es consciente de su dependencia económica. El hecho de que estén altamente de acuerdo con los impactos negativos y positivos planteados en los cuestionarios usados ha llevado a algunos autores a denominarlos "Realists" (Fredline y Faulkner, 2000; Madrigal, 1995). El estudio de Fredline y Faulkner (2000) encuentra paralelismos entre los grupos Haters, Lovers y Realists de Gold Coast y del estudio de Madrigal (1995).

En las regiones con un desarrollo turístico escaso o con amplias zonas exentas de presencia turística se detectan grupos más o menos grandes de personas que muestran respuestas confusas. Estas respuestas confusas ocultan en muchos casos una falta de conocimiento de primera mano del sector debido a la falta de contacto con el mismo. Esto puede deberse a que aún es poco presente en la región o a que el individuo en concreto vive alejado de las zonas más turísticas y tiene poco contacto con los visitantes.

Finalmente cabe destacar que en algunos estudios se detecta un grupo de individuos que se caracterizan por responder en clave personal y no social como en el resto de grupos. Son personas que valoran el desarrollo turístico en relación a lo que reciben y dan ellos a nivel individual y no tienen en cuenta los efectos globales sobre la sociedad o la región. En muchos casos son personas recién llegadas a la región para trabajar en el sector y con poca integración en la comunidad local.

La repetición de ciertas pautas en los distintos estudios lleva a aventurar que pueden existir tipologías preestablecidas de residentes cuyo peso en la sociedad dependerá del destino concreto objeto de estudio.

\section{Segmentación teórica}

Tras revisar diversos estudios en los que se realiza una segmentación de los residentes según su actitud hacia el turismo y observar ciertos paralelismos, parece lógico intentar establecer una segmentación teórica que permita plasmar las distintas tendencias existentes en una región. Esta segmentación teórica dividiría la población en cinco grupos (Figura 1): Partidarios Entusiastas, Partidarios con Opinión Matizada, Partidarios Interesados, Críticos y Sin Opinión Formada.

- Partidarios Entusiastas (hasta el 45\%, normalmente entre el 10\% y el 25\%). Este grupo de opinión existe a lo largo de todo el ciclo de vida del destino y parece que ronda entre el $10 \%$ y el $20 \%$ de la población en destinos maduros y masificados y entre el $20 \%$ y el $30 \%$ en destinos en fases de desarrollo más incipientes. Puede ser muy numeroso ante posibilidades de desarrollo especialmente atractivas. Este grupo son las personas con una opinión más favorable, ya que valoran enormemente los impactos positivos e infravaloran los impactos negativos del turismo. Tienden a perder peso en la sociedad al avanzar el desarrollo en favor de los Partidarios con Opinión Matizada y los Críticos. Este grupo está formado por las personas que reciben, o esperan recibir, de una forma más directa los impactos positivos del desarrollo turístico, por ejemplo trabajadores y empresarios del sector con altas rentas.

- Partidarios con Opinión Matizada (entre el 20\% y el 60\%). Este grupo puede no estar presente en los inicios, pero aumenta su presencia con el desarrollo y se encuentra ampliamente vinculado a la dependencia turística que posea la región. Alcanza su máximo peso en regiones con un desarrollo muy fuerte y una elevada dependencia económica. Las opiniones expresas por este grupo son positivas pero matizadas por el reconocimiento de los impactos negativos. En líneas generales su actitud es positiva, pero se debe a que reconocen la dependencia personal y regional que existe hacia los ingresos generados por el turismo.

- Partidarios Interesados (entre el 10\% y el 35\%). Este grupo es insignificante en los inicios y crece al aparecer flujos de inmigración generados por la falta de mano de obra. En este caso se trata de personas que apoyan el turismo por intereses personales, ya que el turismo les aporta los ingresos económicos que les llevó hasta la región. Suelen ser personas inmigrantes llegadas por motivos económicos a la región que llevan un corto periodo de tiempo residiendo en la región y no tienen apego a la comunidad local. Posiblemente, en el futuro abandonen la región.

- Críticos (hasta el 40\%, normalmente entre el 10\% y el 25\%). Las personas críticas con el turismo suelen rondar el 10\% de la población, pero puede variar en función de elementos culturales o sociales de la población local y del nivel de desarrollo y colapso turístico que viva la región, llegando a porcentajes muy elevados en casos extremos. Suelen ser personas que no perciben de forma directa los beneficios que aporta el turismo, pero si los costes, y que consideran muy grave el cambio cultural y la pérdida de capacidad de decisión sobre el futuro de la región.

- Sin Opinión Formada (entre 0\% y 60\%). En el inició del desarrollo turístico este grupo es el mayoritario, pero con el paso del tiempo va reduciéndose debido a que la población incrementa continuamente su implicación en el sector turístico. En los destinos maduros este grupo es inexistente o insignificante al existir un enorme conocimiento del sector que convierte a estos individuos en Críticos o 
Partidarios, en sus diversas vertientes. Aun así, puede existir un grupo de personas con poco interés en el sector, poco apego a la comunidad, baja dependencia económica del turismo y opiniones poco formadas (los Neutros o Moderados), en los destinos consolidados. Generalmente la presencia de Neutros o Moderados en un destino consolidado se debe a la presencia de zonas o sectores económicos totalmente independientes del turístico. Estas zonas o sectores representan en muchos casos islas de la comunidad previamente existente.

Figura 1: Posicionamiento de los distintos segmentos teóricos.

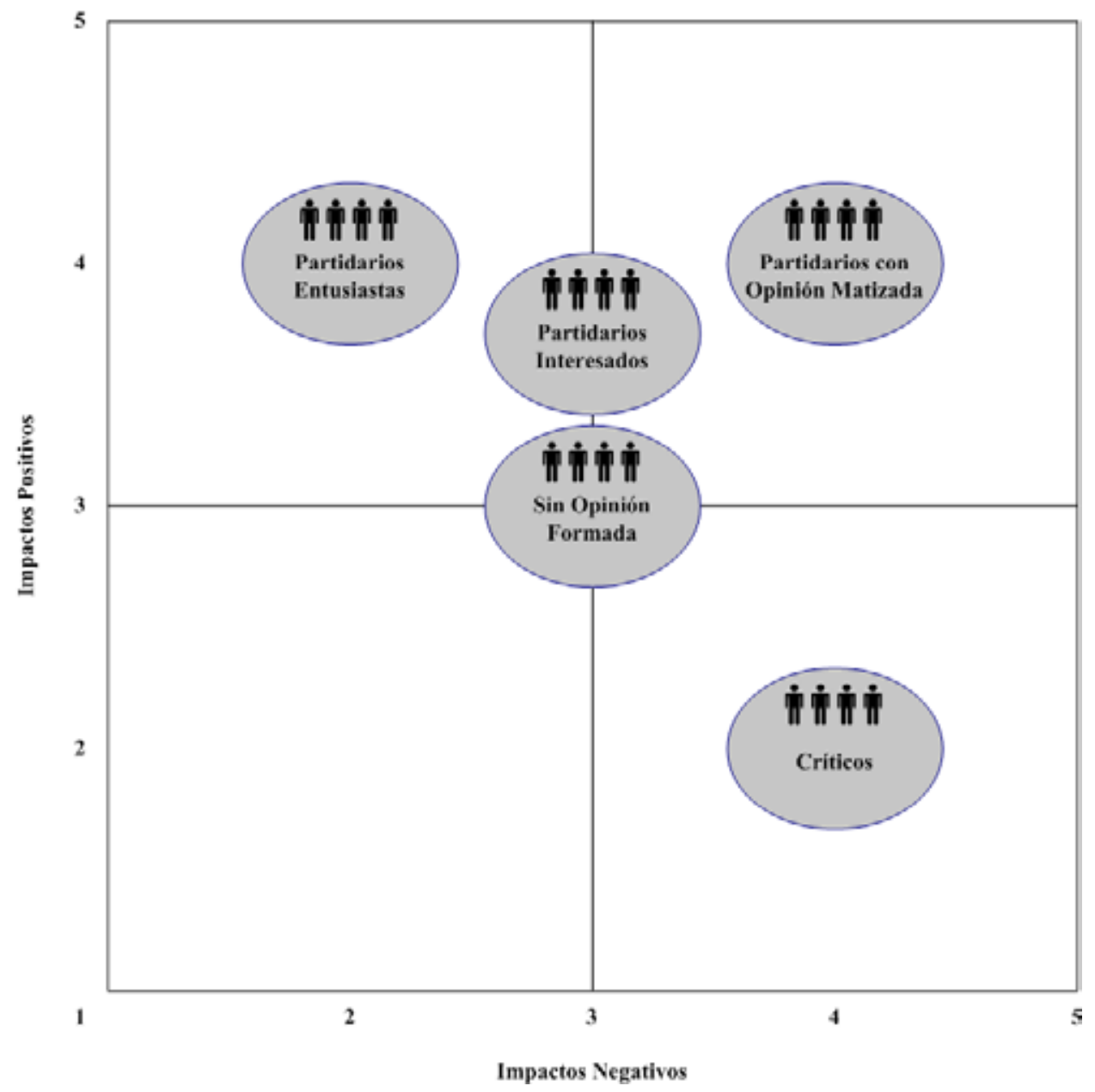

Fuente: Elaboración propia

No tienen porque aparecer en todas las regiones los cinco grupos. Si estarán los Partidarios Entusiastas y lo Críticos, como vemos que se repite en los diversos estudios empíricos. Pero los otros tres grupos oscilarán enormemente o incluso desaparecerán en según qué casos. Los individuos Sin Opinión Formada serán la inmensa mayoría en los inicios del turismo, junto con un grupo de Partidarios Entusiastas que actuaran de motor de desarrollo y una pequeñísima presencia de Críticos.

En las primeras fases de desarrollo turístico los individuos Sin Opinión Formada irán integrándose en los Partidarios Entusiastas o los Partidarios con Opinión Matizada, según lo beneficioso que resulte 
para ellos la implicación en el sector. Las personas que resulten marginadas de los beneficios generados y que sufran los costes pasarán a integrar el grupo de los Críticos.

En la fase de madurez los Partidarios Entusiastas representan una parte pequeña de la población. Si el nivel de dependencia de la región es muy elevado el grupo mayoritario será el de los Partidarios con Opinión Matizada, desapareciendo el grupo de los Sin Opinión Formada. Si el desarrollo no es tan intenso o el turismo se combina con otros sectores económicos, los Partidarios con Opinión Matizada serán menos numerosos y permanecerá un grupo significativo de Neutros o Moderados como supervivencia de los individuos Sin Opinión Formada.

Dependiendo de la planificación y grado de masificación que sufra la región en su evolución, será mayor o menor el grupo de los Críticos. Si el desarrollo ha sido planificado y toda la población resulta beneficiada, el grupo de los Críticos tendrá una presencia mínima y los impactos negativos se plasmarán en la fuerte presencia de Partidarios con Opinión Matizada. En cambio, si el desarrollo es descontrolado, tiende a la masificación, causa fuertes impactos negativos y hay un mal reparto de los beneficios, el grupo de los Críticos será muy significativo. Si los Críticos superan a los Partidarios, en sus distintas modalidades, el destino turístico llegará a su fin como tal ya que las presiones para reducir o eliminar este sector superaran a las presiones para mantenerlo.

Esta evolución se produciría de forma parecida a lo que muestran los modelos Irridex de Doxey (1975) y GIPM de Vong y McCartney (2005), pero con el matiz de que en este caso en todo momento hay varios grupos interactuando en la región.

\section{Conclusiones}

En las últimas décadas se han realizado diversos estudios en los que se realizaba una segmentación de los residentes de un destino turístico que pretendía agruparlos según sus actitudes hacia el turismo y sus impactos. Estos estudios poseen ciertas variaciones en la segmentación resultante, pero si se comparan pueden observarse ciertas pautas que se repiten. Se observan grupos siempre presentes y grupos que aparecen y desaparecen según el caso. Esto lleva a plantear la posibilidad de establecer una segmentación teórica que permita obtener una visión general.

En la segmentación teórica planteada se describen cinco grupos: los Partidarios Entusiastas, los Partidarios con Opinión Matizada, los Partidarios Interesados, los Críticos y los Sin Opinión Formada. De estos cinco grupos los Partidarios Entusiastas y los Críticos siempre estarán presentes en los estudios empíricos que se realicen, aunque puede que sea en porcentajes muy pequeños, y los otros tres grupos aparecerán o desaparecerán, crecerán o disminuirán dependiendo de las características del destino y del tamaño de la muestra que se tome. Si la muestra analizada es excesivamente pequeña existe el riesgo de que sólo aparezcan tres grupos: Partidarios, Críticos e Indefinidos.

Más allá de su uso como posible hipótesis para trabajos empíricos la principal utilidad de esta segmentación es servir de modelo teórico a los gestores de los distintos destinos turísticos para mejorar sus acciones dirigidas a los residentes, ya que aunque no se conozca el peso de cada grupo en un destino concreto si se sabe que el grupo está o puede estar. En general, los gestores deben tomarse acciones encaminadas a aumentar los beneficios que obtienen los residentes (desarrollo autóctono de la oferta, oferta con elevados estándares de calidad, infraestructuras y servicios aprovechables por los residentes y los turistas, etc.), reducir los impactos negativos (inseguridad, ruidos, degradación del entorno, etc.) e implicar a los residentes en la gestión turística (informándolos y fomentando su participación en la toma de decisiones). Cabe tener presente que no hay desarrollo posible si los grupos de Partidarios no superan en número a los Críticos, y las acciones mencionadas deben buscar evitar que los individuos Sin Opinión Formada o Partidarios del turismo pasen a ser Críticos al evolucionar el destino a lo largo del Ciclo de Vida del Destino Turístico.

De cara al futuro se debería analizar la interacción de esta clasificación con dos parámetros que suelen resultar significativos en estos estudios: el tiempo de residencia en la región (Aguiló y Rosselló, 2005; Brida, Osti y Barquet, 2010; Ryan y Montgomery, 1994; Weaver y Lawton, 2001; Williams y Lawson, 2001) y si el individuo es nativo o no (Aguiló y Rosselló, 2005; Canan y Hennessy, 1989; Davis, Allen y Cosenza, 1988; Evans, 1993; Madrigal, 1995; Um y Crompton, 1987). Estos dos elementos permitirían pasar de la clasificación actual a nuevas dimensiones de desglose que permitan un mayor detalle de la población residente. 


\section{Bibliografía}

Aguiló, E. y Rosselló, J.

2005 "Host Community Perceptions. A Cluster Análisis". Annals of Tourism Research, 32(4): 925-941. Aguiló, E., Barros, V., García, M. A. y Rosselló, J.

2004 Las actitudes de los residentes en Baleares frente al turismo. Turisme i Investigació ${ }^{\circ} 7$. Palma de Mallorca: Universitat de les Illes Balears.

Allen, L. R., Hafer, H. R., Long, P. T. y Perdue, R. R.

1993 "Rural residents' attitudes toward recreation and tourism development". Journal of Travel Research, 32(1): 27-33.

Allen, L. R., Long, P. T., Perdue, R. R. y Kieselbach, S.

1988 "The impact of tourism development on residents' perceptions of community life". Journal of Travel Research, 27(1): 16-21.

Andriotis, K. y Vaughan, R. D.

2003 "Urban residents' attitudes toward tourism development: The case of Creta". Journal of Travel Research, 42(2): 172-185.

Besculides, A., Lee, M. y McCormick, P.2002 "Resident's perceptions of the cultural benefits of tourism". Annals of Tourism Research, 29(2): 303-319.

Brida, G. G., Osti, L. y Barquet, A.

2010 "Segmenting Resident Perceptions towards Tourism - a Cluster Analysis with a Multinomial Logit Model of a Mountain Community". International Journal of Tourism Research, 12(5): 591-602.

Brougham, J. E.

1978 Resident attitudes towards the impact of tourism in Sleat. Unpublished Ph.D. thesis, University of Western Ontario (Canada).

Brougham, J. E. y Butler, R. W.

1977 The social and cultural impact of tourism: A case study of Sleat, Isle of Skye. Edinburgh: Scottish Tourist Board.

1981 "A segmentation analysis of resident attitudes to the social impact of tourism". Annals of Tourism Research, 8(4): 569-590.

Brownrigg, M. y Greig, M. A.

1976 Tourism and regional development. Glasgow: Fraser of Allander Institute.

Butler, R. W.

1980 "The concept of a tourist area cycle of evolution: Implications for the management of resources". The Canadian Geographer, 24(1): 5-12.

Canan, P. y Hennessy, M.

1989 "The growth machine, tourism and the selling of culture". Sociological Perspectives, 32: 227-243.

Davis, D., Allen, J. y Cosenza, R. M.

1988 "Segmenting local residents by their attitudes, interests and opinions toward tourism". Journal of Travel Research, 27(2): 2-8.

Díaz, R., Gutiérrez, D. y Garau, J.

2007 "Actitud del Residente en Destinos Turísticos Insulares: Un Análisis Comparativo”. XIX Encuentro de Profesores Universitarios de Marketing.

Doxey, G. V.

1975 "A causation theory of visitor-resident irritants: methodology and research inferences". En Conference Proceedings: Sixth Annual Conference of Travel and Tourism Research Association (San Diego), pp. 195-198.

Evans, T. R.

1993 Resident's perceptions of tourism in selected New Zealand communities: a segmentation study. Unpublished Master thesis. Dunedin: Dept. of Commerce, University of Otago.

Fredline, E. y Faulkner, B.

2000 "Host community reactions: A cluster analysis". Annals of Tourism Research, 27(3): 763-784.

Gursoy, D., Chen, J. S. y Yoon, Y.

2000 "Using structural equation modeling to assess the affects of tourism impact factors and local residents support for tourism development". Annual conference proceedings of the Travel and Tourism Research Association (Boise), pp. 243-250.

Gursoy, D., Jurowski, C. y Uysal, M.

2002 "Resident attitudes: a structural Modeling Approach". Annals of Tourism Research, 29(1): 79-105. Gutiérrez, D. y Díaz, R.

2006 "Actitud del Residente en un Destino Complejo y Diverso: Inestabilidad o Consistencia". XVIII Encuentro de Profesores Universitarios de Marketing. 
Jurowski, C., Uysal, M. y Williams, R. D.

1997 "A theoretical analysis of host community resident reactions to tourism". Journal of Travel Research, 36(2): 3-11.

Keogh, B.

1990 "Public participation in community tourism planning". Annals of Tourism Research, 17(3): 449-465.

Lankford, S. V.

1994 "Attitudes and perceptions toward tourism and rural regional development". Journal of Travel

Research, 24(3): 35-44.

Lankford, S. V. y Howard, D. R.

1994 "Developing a tourism impacts attitude scale". Annals of Tourism Research, 21(1): 121-139.

Lawson, R. W. y Todd, S.

1997 "Relationships between Consumer Sentiment towards Marketing and Consumer Lifestyles". En

Falkenberg, A. W. y Rittenberg, T. L. (edi), $22^{\text {nd }}$ Annual MacroMarketing Conference (Vol. 1). Bergen:

Norges Handelshoyskole, Intitutt for Markedsokonomi.

Madrigal, R.

1995 "Residents' perceptions and the role of government". Annals of Tourism Research, 22(1): 86-102.

Martin, S.

1995 "Montanans' attitudes and behavioral intentions toward tourism: Implications for sutainability".

En McCool, S. y Watson, A. (Eds.), Linking Tourism, the Environment, and Sustainability. Ogden

UT: US Department of Agriculture, Forest Service, pp. 69-76.

Mason, P. y Cheyne, J.

2000 "Resident's attitudes to proposed tourism development". Annals of Tourism Research, 27(2): 391-411. Murphy, P. E.

1985 Tourism: A community approach. New York: Routledge.

Perdue, R. R., Long, P. T. y Allen, L.

1990 "Resident support for tourism development". Annals of Tourism Research, 17(4): 586-599.

Pi-Sunyer, O.

1977 "Through native eyes: Tourists and tourism in a catalan maritime community". En Smith, V.

(Edi.), The Anthropology of Tourism. Philadelphia: University of Pennsylvania Press, pp. 149-155.

Ryan, C. y Montgomery, D.

1994 "The attitudes of bakewell residents to tourism and numbers in comunity responsive tourism".

Tourism Management, 15(5): 358-369.

Ryan, C., Scotland, A. y Montgomery, D.

1998 "Resident attitudes to tourism development-A comparative study between the Rangitikei, New

Zealand and Bakewell, United Kingdom”. Progress in Tourism and Hospitality Research, 4(2): 115-30.

Schewe, C. D. y Calantone, R. J.

1978 "Psychographic segmentation of tourists". Journal of Travel Research, 16(3): 14-20.

Schroeder, T.

1992 "Host Community Perceptions of Tourism's impacts: A cluster analysis". Visions in Leisure and

Business, 10(4): 43-48.

Teye, V., Sirakaya, E. y Sönmez, S.

2002 "Resident's attitudes toward tourism development". Annals of Tourism Research, 29(3): 668-688. Thyne, M. y Lawson, R.

2001 "Research Note: Addressing tourism public policy issues through attitude segmentation of host

communities". Current Issues in Tourism, 4(2-4): 392-400.

Um, S. y Crompton, J. L.

1987 "Measuring resident's attachment levels in a host community". Journal of Travel Research, 26(1): 27-29.

Vong, F. y McCartney, G.

2005 "Mapping resident perceptions of gaming impact". Journal of Travel Research, 44(2): 177-187.

Weaver, D. y Lawton, L.

2001 "Resident perceptions in the urban-rural fringe". Annals of Tourism Research, 28(2): 439-458.

Williams, J. y Lawson, R.

2001 "Community issues and resident opinions of tourism". Annals of Tourism Research, 28(2): 269-290.

Recibido:

$02 / 05 / 2013$

Reenviado:

$29 / 07 / 2013$

Aceptado:

$09 / 09 / 2013$

Sometido a evaluación por pares anónimos 